\title{
Accurate Characterization of Multi-Resonant Reflectarray Cells by Artificial Neural Networks.
}

\author{
P. Robustillo , J. Zapata, J.A. Encinar, R. Florencio , R. R. Boix , J.R. Mosig
}

\begin{abstract}
This paper describes the accurate characterization of the reflection coefficients of a multilayered reflectarray element by means of artificial neural networks. The procedure has been tested with different RA elements related to actual specifications. Up to 9 parameters were considered and the complete reflection coefficient matrix was accurately obtained, including cross polar reflection coefficients. Results show a good agreement between simulations carried out by the Method of Moments and the ANN model outputs at RA element level, as well as with performances of the complete RA antenna designed.
\end{abstract}

Index Terms-reflectarray, artificial neural networks.

\section{INTRODUCTION}

In recent years, a growing interest in reflectarray antenna has arisen, [1]. Reflectarrays (RA) are planar structures made up of hundreds or thousands of small single elements, called as RA elements. Among its virtues, we find their capabilities to produce contoured beams and the ease of fabrication compared to shaped reflectors. However, RA design is still slow since many calls to electromagnetic (EM) solver routines are needed in the optimization process. Even though some techniques have been implemented to make EM methods faster [2], [3], they are still slow. This effect is more severe when the number of free geometrical parameters in each RA element is high. This latter requirement is especially necessary when multi frequency design is required [4].

One important technique to be used for RA element optimization instead of calls to EM procedures is the use of Artificial Neural Networks, [5]. ANNs are considered in this EM context as multiple-input, multiple-output, non-linear interpolators, [6]. Before getting ready to interpolate, ANNs must be trained. For the training process, a set of input vectors and their corresponding EM outputs are needed. By using this training set, ANNs learn how to interpolate for inputs within the range of interest.

In [7], the ANN characterization of a double-ring RA element was carried out. Co-polar reflection coefficient was modeled as a function of the incidence angle and a geometrical free parameter. Similar characterizations were carried out in [8] and [9]. This work presents an accurate characterization of a multi-resonant RA element, considering the co-polar components of the reflection coefficients as well as the crosspolar components, for amplitude and phase. The number of free parameters is 9: six geometrical free parameters, the two coordinates of the incidence angle and the frequency.

\section{ACCURATE REFLECT ARRAY ELEMENT CHARACTERIZATION}

\section{A. Geometrical Parameters}

The kind of RA element considered in this paper is a multilayered structure, made up of dielectric layers where metallic pieces (i.e. dipoles, patches, etc.) are stacked. This is a very usual structure for RA element, since it can show wide band-width by properly adjusting the dimensions of its metallic pieces, [4]

In order to obtain the training set, each potential input parameter (i.e. geometrical parameter, incidence angle, frequency, etc.) must be swept from its minimum value to its maximum value. These border values, as well as the step size, determine the size of the discretized input set. We can profit from the multilayer structure, [5], [10]. Thanks to it, the speed up factor for training set computation would be about $30-50$.

\section{B. Incidence Angle}

Since RA elements are spread anywhere on the reflector surface, every RA element sees the incidence wave coming from a different direction. That is why the incidence angle is also considered as an input parameter when characterizing RA elements. This is particularly important when an accurate modeling is required, including amplitude and phase of both co- and cross-polar components, in order to design a high performance reflectarray antenna.

\section{ARTIFICIAL NEURAL NETWORK DEFINITION}

In this paper, we consider Multilayer Perceptron Artificial Neural Networks (MLP ANN), [6]. Three hidden layers are considered, with 20 neurons each.

We considered a different ANN for every output. Complex values were considered as two different values: real part and imaginary part. We also considered a different ANNs for every value of the incidence angle in the training set. 


\section{RESULTS}

Following the guideline described above, the RA element presented in Fig. 1 has been characterized.

The RA element is made up of two dielectric layers. On each layer, three metallic dipoles were placed. The length of each dipole was considered as a free geometrical parameter. The whole input set ranges are:

$$
\begin{aligned}
& l_{A 1}=(0.5-0.65) l_{A 2}, 4 \text { values } \\
& l_{A 2}=5.5-11 \mathrm{~mm}, 50 \text { values } \\
& l_{A 3}=l_{A 1} \\
& l_{B 1}=(0.5-0.65) l_{B 2}, 4 \text { values } \\
& l_{B 2}=5.5-11 \mathrm{~mm}, 50 \text { values } \\
& l_{B 3}=l_{B 1} \\
& \theta=0^{\circ}, 10^{\circ}, 20^{\circ} \\
& \varphi=0^{\circ}-180^{\circ}, \Delta \varphi=15^{\circ}
\end{aligned}
$$

\section{$f=11.95 \mathrm{GHz}$}

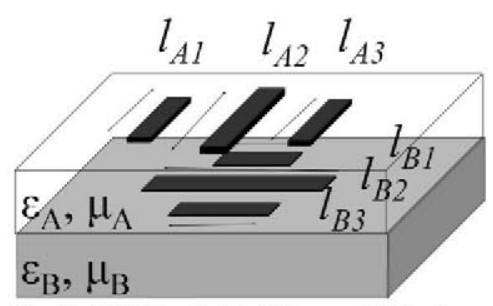

Fig. 1. 3+3 Dipole Multi-layered RA element.

The total number of samples was 40.000 per incidence angle. The total time to obtain the complete training set was 4.5 hours, where parallelization in the frequency values was carried out.

Since the most difficult parameters to be accurately modeled are the cross-polar components of the reflection coefficient, Figures 2 and 3 show the amplitude and phase of the YX cross polar component of the reflection coefficient matrix. MoM results, in blue, are superimposed to ANN outputs, red, for the same values of the input vectors. A good agreement is observed. Similar results were obtained for the rest of components and incidence angles.

To check out the accuracy of the ANN in both co-and cross-polar components, a reflectarray antenna has been designed. The RA antenna met the requirements given in [5]. The central band frequency is $11.95 \mathrm{GHz}$, and the coverage area is the south American region.

Figure 4 shows the gain patterns for co polar and cross polar. The antenna designed by using the ANN met the specifications, however with design time for a single frequency was 103 seconds in a PC with Intel Xeon 5150 processor, at $2.66 \mathrm{GHz}$ and $6 \mathrm{~GB}$ of RAM whereas designing a similar antenna under the same conditions by MoMs is a question of hours.

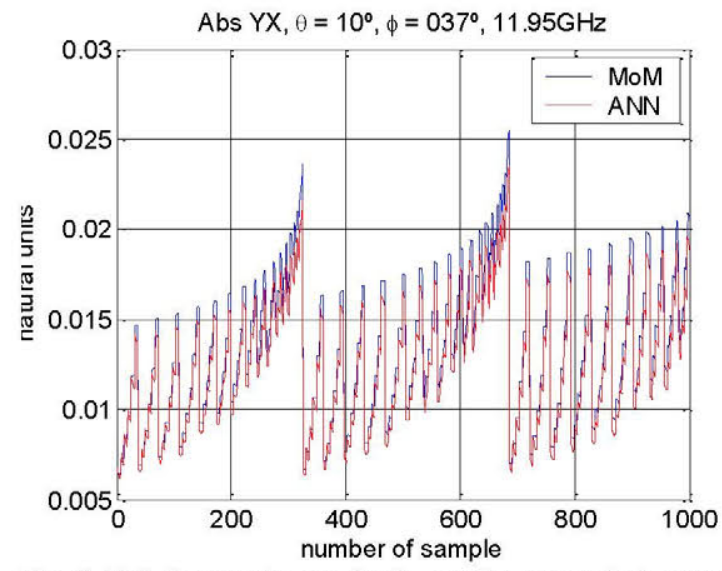

Fig. 2. YX Cross polar amplitude. X-axis: geometrical parameter values; yvalue: natural units. Blue: MoM; Red: ANN.

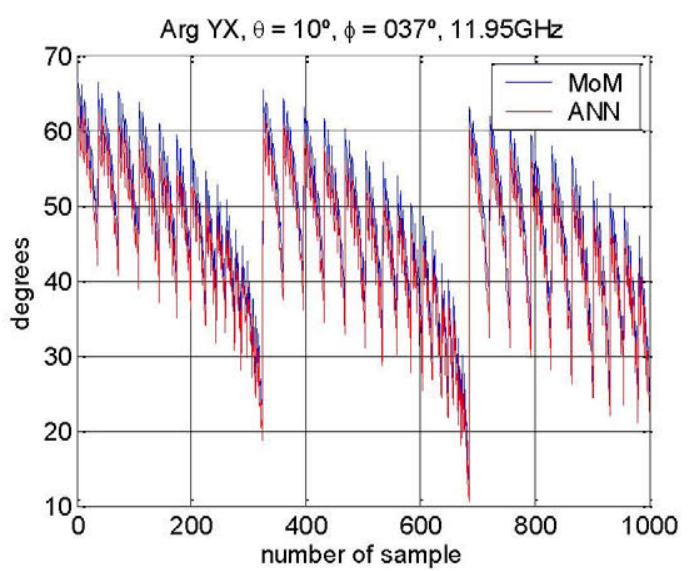

Fig. 3. XY Cross polar phase. x-axis: geometrical parameter values; $y$-value: degrees. Blue: MoM; Red: ANN.

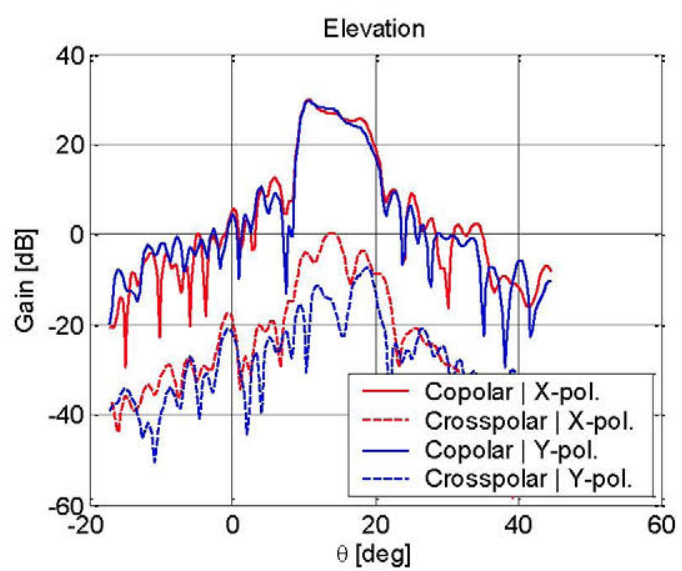

(a) 


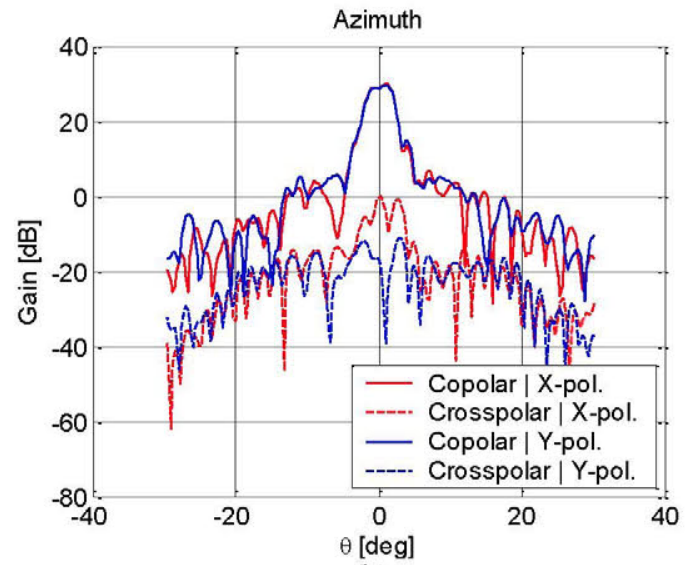

(b)

Fig. 4. RA design by ANN. Co polar/cross polar gain patterns. Cuts at principal planes.

\section{CONCLUSIONS}

This paper shows an accurate ANN characterization of a multi-resonant reflectarray cell. It is remarkable the good accuracy obtained in predicting the cross polar components of the reflection coefficients. This topic has barely been taken into account in the RA literature even though it is a very promising future line for cross polar reduction in RA design.

\section{ACKNOWLEDGMENT}

This work has been partially supported by the Spanish Government under projects TEC2010-20249-C02-01, CICYT TEC2010-17567, CONSOLIDER CSD2008-00068, TEC2010-20249-C02-01, and by the European Space Agency, under project ESTEC ITT AO/1-7064/12/NL/MH.

\section{REFERENCES}

[1] J. Huang, J. A. Encinar, Reflectarray Antennas, Piscataway, NJ: IEEE Press, 2008.

[2] P. Gay-Balmaz, J. A. Encinar, J. R. Mosig, "Analysis of multilayer printed arrays by a modular approach based on the generalized scattering matrix", IEEE Transactions on Antennas and Propagation, vol. 48 , pp. $26-34,2000$.

[3] W. Changhua, J. A. Encinar, "Efficient computation of generalized scattering matrix for analyzing multilayered periodic structures", IEEE Transactions on Antennas and Propagation, vol. 43, pp. 1233-1242, 1995.

[4] J. A. Encinar, M. Arrebola, L. F. de la Fuente, G. Toso, "A transmitreceive reflectarray antenna for Direct Broadcast Satellite Applications", IEEE Transactions on Antennas and Propagation, vol. 59, pp. 3255-3264, 2011.

[5] P. Robustillo, J. Zapata, J.A. Encinar, J. Rubio, "ANN Characterization of Multi-Layer Reflectarray Elements for Countoured-Beam Space Antennas in the Ku-Band", IEEE Transactions on Antennas and Propagation, vol. 60, pp. 3205 -3214, July 2012.

[6] S. Haykin, Neural networks, a Comprehensive Foundation, $2^{\text {nd }}$ Ed., Englewood Cliffs, NJ: Prentice-Hall.

[7] M. Mussetta, P. Pirinoli, R. E. Zich, M. Orefice, "ANN characterization of printed reflectarray elements", in Proc. IEEE Antennas and Propagation Society International Symposium (APSURSI), 2010.

[8] M. Mussetta, P. Pirinoli, P. T. Cong, M. Orefice, R. E. Zich, "Characterization of microstrip reflectarray square ring elements by means of an Artificial Neural Network", in Proc. 4th European Conference on Antennas and Propagation, 2010.

[9] S. Nesil, F. Gunes, U. Ozkaya, "Phase characterization of a reflectarray unit cell with Minkowski shape radiating element using Multilayer Perceptron Neural Network", in Proc. 7th International Conference on Electrical and Electronics Engineering, 2011.

[10] R. Florencio, R.R. Boix, E. Carrasco, J. A. Encinar, V. Losada, "Efficient Numerical Tool for the Analysis and Design of Reflectarrays Based on Cells with Three parallel dipoles", Microwave and Optical Tech. Letters, vol. 55, no. 6, June 2013. 